\title{
Concept for a MEMS-type vacuum sensor based on electrical conductivity measurements
}

\author{
Friederike Julia Giebel $^{1}$, Marcel Köhle ${ }^{2}$, Till Stramm ${ }^{2}$, Klaus T. Kallis ${ }^{1}$, and Horst L. Fiedler ${ }^{2}$ \\ ${ }^{1}$ Micro- and Nanoelectronic Devices, Faculty of Electrical Engineering and Information Technology, \\ TU Dortmund University, Dortmund 44227, Germany \\ ${ }^{2}$ Intelligent Microsystems Institute, Faculty of Electrical Engineering and Information Technology, \\ TU Dortmund University, Dortmund 44227, Germany
}

Correspondence to: Friederike Julia Giebel (friederike.giebel@tu-dortmund.de)

Received: 30 September 2016 - Revised: 25 September 2017 - Accepted: 29 September 2017

- Published: 16 November 2017

\begin{abstract}
The concept of the micro-structured vacuum sensor presented in this article is the measurement of the electrical conductivity of thinned gases in order to develop a small, economical and quite a simple type of vacuum sensor. There are already some approaches for small vacuum sensors. Most of them are based on conservative measurement principles similar to those used in macroscopic vacuum gauges. Ionization gauges use additional sources of energy, like hot cathodes, ultraviolet radiation or high voltage for example, for ionizing gas molecules and thereby increasing the number of charge carriers for measuring low pressures. In contrast, the concept discussed here cannot be found in macroscopic sensor systems because it depends on the microscopic dimension of a gas volume defined by two electrodes. Here we present the concept and the production of a micro-structured vacuum sensor chip, followed by the electrical characterization. Reference measurements with electrodes at a distance of about $1 \mathrm{~mm}$ showed currents in the size of picoampere and a conductivity depending on ambient pressure. In comparison with these preliminary measurements, fundamental differences regarding pressure dependence of the conductivity are monitored in the electrical characterization of the micro-structured sensor chip. Finally the future perspectives of this sensor concept are discussed.
\end{abstract}

\section{Introduction}

The demand for miniature pressure sensors is increasing steadily due to various kinds of application. New fields of application are established because of miniaturization of sensors and sensor systems. Additionally, smaller devices and cost-effective production cause lower unit prices. One area of application is low pressure measurement. Measuring instruments for ultra-high vacuum are usually needed in fields of highly specialized industry or research, for example in scanning electron microscopes or particle accelerators; whereas measurement devices for low to high vacuum are employed in broad fields of industry, from automotive sensor applications to food processing. Even in the fabrication of microelectro-mechanical systems (MEMS), vacuum is needed to increase reliability and endurance (Górecka-Drzazga, 2009).
Due to the growth of the market for micro-structured vacuum sensors there are already many approaches for fabricating such devices. One popular idea is the miniaturization of Pirani gauges that use the dependence of thermal conductivity on ambient pressure (Wang et al., 2010; Dankovic et al., 2012). The concepts for micro-Pirani gauges have been improved for decades and in the meantime MEMS-type gauges with a very wide pressure range from $10^{-5}$ to $10^{3}$ mbar have been presented (Zhang et al., 2006; Völklein and Meier, 2007; Sun et al., 2013).

It is not surprising that measuring the electrical conductance of thinned gaseous substances has not been of interest for low pressure measurements so far. In general miniaturization of well-known sensor types is the typical approach regarding the development of microsensors and most MEMStype vacuum sensors make use of effects that are known from 
conservative, macroscopic gauges (Tenholte et al., 2008). Some newer concepts of micro-structured vacuum gauges, based on field emission, were established during the last few years. Even though there is skepticism about whether or not the miniaturization of ionization vacuum gauges will be advantageous, some approaches with field emitter arrays have been presented (Górecka-Drzazga, 2009; Wilfert and Edelmann, 2012). One concept for pressure sensors operates with the effect of field emission from silicon tip arrays. A difficulty here was the need for high sensitivities that seems to exclude the need for high currents (Lee and Huang, 1991; Wen et al., 2007).

In general, gases are considered to be very good insulators. On a macroscopic scale the electrical current through a gas volume is very small. To measure these currents a high level of measurement technique is necessary: digital multimeters are usually suitable for measuring currents greater than $1 \mu \mathrm{A}$. For low current measurements, picoammeters are required, triaxial cables must be used to achieve a good guarding of the signals and the overall measurement setup must also be shielded from electromagnetic interference in order to minimize the noise level (Zhou et al., 2012; TEKTRONIX Inc., 2013). That is why this sensor principle is not attractive on a macroscopic scale. The manufacturing of a gas chamber with electrodes at a distance of some $100 \mathrm{~nm}$ provides a chance to use electrical conductivity measurements for the measurement of low pressure. Even if gases are considered to be very good insulators, there is always a small number of charge carriers depending on the gas pressure. The miniaturization of the MEMS-type gas chamber leads to an increase in the electrical conductance of the device and thus to an increase in the currents to be measured. This reduces the requirements of measurement technique and opens up an interesting new measurement concept for the determination of vacuum pressures.

This study aims to examine whether conductivity measurements with MEMS devices are a promising concept for simple and competitive microscopic sensors for low pressure measurements. In the following, some theoretical background concerning the principle of electrical conductance in gases will be summarized. The design, the fabrication in standard CMOS technology and the electrical characterization of the sensor chip will be explained afterwards. Finally, the results of the electrical characterization will be presented, analyzed and discussed.

\section{Conductivity in gases}

Assuming that the number of charge carriers in a gas volume is a function of the total number of particles in that volume and thereby a function of pressure, the measurement of the electrical conductance $G$ of a gas volume is a way to determine the gas pressure. Measuring the current-voltage characteristics is general practice to determine the electrical con- ductance $G$, which is the derivative

$G=\left.\frac{d I}{d U}\right|_{U}$

of the current $I$ with respect to the voltage $U$. The challenge of measuring electrical conductances in hardly conductive materials is the low current measurement. Therefore, the sensitivity of the experimental setup is crucial for measuring conductivities of gases. The conductance of a resistive volume will increase with the area perpendicular to the electric field $A$ and decrease with the length of the gas volume, or more precisely the distance between the two electrodes $d$. Thus, a gas volume of a small length and large area, that can be realized by the application of surface micro-machining technology, will provide enlarged conductances and currents.

Certainly, the current-voltage characteristics of two electrodes with more or less gas molecules in-between cannot be described by Ohm's law. First of all, the electrical conductivity $\kappa$ may be a function of the electric field. In addition, the total current is affected by diffusion and the source of charge carriers. For understanding the behavior of the sensor chip, it is necessary to examine the conduction mechanisms that might take place. Two special cases shall be discussed in the following paragraphs. In the first case, a number of gamma electrons are emitted from a cathode per unit area and time. The constant emission current density is causing an emission-limited current flow (Rutherford and Goldston, 1995). The electrons released from the cathode either drift to the anode due to the electric field $E$ or return to the cathode by diffusion with the thermal mean speed $\langle u\rangle$. Therefore the sum of the current densities,

$\gamma e-\rho \mu E-\frac{\rho\langle u\rangle}{4}=0$,

equals zero, where $e$ is the elementary charge, $\rho$ is the charge density and $\mu$ is the electrical mobility of the charge carriers. The current between cathode and anode results in

$I_{\text {cath. }}=\frac{\gamma e A}{\left(\mu U+\frac{d\langle u\rangle}{4}\right)} \mu U$,

where $d$ is the distance between those two electrodes, $A$ is the area perpendicular to the electric field $E$ and $U$ is the voltage across the electrodes.

In the second special case, the charge carriers originate from ionization in the volume between two electrodes. Some charge carriers generated diffuse towards the edge of the volume and some of them drift towards the electrodes. Neglecting recombination, in equilibrium the number of charge carriers $N$ does not change over time and can be described by

$\left(\frac{\mathrm{d} N}{\mathrm{~d} t}\right)=n_{\mathrm{g}} \nu_{0} V-\frac{N}{\tau}-\frac{2 N|\langle\boldsymbol{u}\rangle|}{d}=0$,

where $n_{\mathrm{g}}$ is the density of the gaseous substance, $v_{0}$ is the frequency of generating charge carriers by ionization, $V$ is the 
volume between the two electrodes, $\tau$ is the mean residence time due to diffusion to the boundaries of the gas volume and $|\langle\boldsymbol{u}\rangle|$ is the average drift velocity in electric field. This results in the total current

$I_{\text {ion. }}=\frac{e \tau \nu_{0} n_{\mathrm{g}} d A}{2 \tau \mu U+d^{2}} \mu U$

between cathode and anode (Wiesemann, 1976).

Ohm's law works for these experimental setups in the case of small electric fields. If $\mu U$ is substantially less than $\frac{d\langle u\rangle}{4}$, Eq. (3) may be approximated by

$$
I_{\text {cath., ohm. }} \approx \frac{4 \gamma e}{\langle u\rangle} \frac{A}{d} \mu U
$$

and if $\mu U$ is substantially less than $\frac{d^{2}}{2 \tau}$, Eq. (5) may be approximated by

$$
I_{\text {ion., ohm. }} \approx e \tau v_{0} n_{\mathrm{g}} \frac{A}{d} \mu U .
$$

Whether these presumptions for a linear approximation are true has to be decided in individual cases.

These characteristics show a pressure-depending behavior as the gas density $n_{\mathrm{g}}$ and the electrical mobility $\mu$ are functions of the pressure. The ideal gas law states that $n_{\mathrm{g}}$ is proportional to the pressure $p$. The electrical mobility $\mu$ is depending on the ratio of electric field to pressure. Wasserrab states that the mobility is proportional to $1 / p$ for small electric fields and high pressures, and that it is proportional to $1 / \sqrt{p}$ for experimental setups with low pressures and higher electric fields (Wasserrab, 1971). For mercury vapor, for example, it is shown that the electron's mobility dependence on the pressure can be described by

$\mu_{-}(p)=\mu_{0-} \cdot \frac{p_{0}}{p}$

with $\mu_{0-}=22.7 \mathrm{~m}^{2} \mathrm{~V}^{-1} \mathrm{~s}^{-1}$ and $p_{0}=1 \mathrm{mbar}$ for an electric field to pressure ratio $E / p$ less than $1.5 \times 10^{4} \mathrm{~V} \mathrm{~m}^{-1} \mathrm{mbar}^{-1}$. The mercury ion's mobility is indicated as

$\mu_{+, 1}(p)=\mu_{0+, 1} \cdot \frac{p_{0}}{p}$,

with $\mu_{0+, 1}=2.4 \times 10^{-2} \mathrm{~m}^{2} \mathrm{~V}^{-1} \mathrm{~s}^{-1}$ for an electric field to pressure ratio $E / p$ less than $7.5 \times 10^{3} \mathrm{~V} \mathrm{~m}^{-1} \mathrm{mbar}^{-1}$ and

$\mu_{+, 2}(p)=\mu_{0+, 2} \cdot \frac{1}{\sqrt{E p}}$,

with $\mu_{0+, 2}=2.1 \mathrm{~m} \mathrm{~s}^{-1}\left(\mathrm{~m} \mathrm{mbar}^{-1}\right)^{1 / 2}$ for an electric field to pressure ratio $E / p$ higher than $7.5 \times 10^{3} \mathrm{~V} \mathrm{~m}^{-1} \mathrm{mbar}^{-1}$.

The combination of Eqs. (6) and (7) with the different parts of mobility (Eqs. 8-10) results in different forms of dependencies between current and pressure, respectively, conductance and pressure. Assuming that the pressure dependence of the conductance is due only to the pressure dependence of the gas density $n_{\mathrm{g}}$ (given by the ideal gas law) and the electrical mobility $\mu$, the following proportionalities can be estimated: in the case of electrons emitted from a cathode, the conductance is proportional to $1 / p$ for $E / p$ less than $1.5 \times 10^{4} \mathrm{~V} \mathrm{~m}^{-1} \mathrm{mbar}^{-1}$. In the case of ionization in the gas volume, the comparison of Eqs. (8) to (10) with Eq. (7) shows that the dependence of the conductance on the pressure at smaller pressures is essentially determined by the mobility of the ions. In this case the pressure dependency of the electrical conductance is given by

$G_{+, 2}(p)=G_{0+, 2} \cdot \sqrt{p / p_{0}}$,

with $p_{0}=1 \mathrm{mbar}$ and the conductance $G_{0+, 2}$ for $p=1 \mathrm{mbar}$ for $E / p$ higher than $7.5 \times 10^{3} \mathrm{~V} \mathrm{~m}^{-1} \mathrm{mbar}^{-1}$. The pressure dependency of the mobility of ions at higher pressures and of electrons cancels the pressure dependence of the gas density in Eq. (7) and then results in a conductance that is independent of pressure.

For the evaluation of the experimental results, a determination of the electric field to pressure ratio is needed. Although the electric field may not be homogeneous, for the experimental setups presented in this article the magnitude of the electric field $E$ may be approximated by

$E \approx \frac{U}{d}$,

where $d$ is the distance between the electrodes.

If the distance between the electrodes $d$ is in the range of the mean free path of electrons or smaller, another effect has to be considered. The mean free path of electrons in kinetic gas theory is given by

$\lambda=\frac{1}{n_{\mathrm{g}} \sigma}$,

where $\sigma$ is the effective cross-sectional area for collision. In perfect vacuum, electrons are accelerated to the anode undamped by collisions and the mobility becomes independent of ambient pressure. With rising pressure, the number of collisions of electrons with gas molecules increases, the mean free path decreases and the fraction of electrons that arrive at the anode without collisions with gas molecules falls off. The pressure dependence of the electrical conductance can then be described by

$G_{\lambda}(p)=G_{0, \lambda} e^{-d / \lambda(p)}=G_{0, \lambda} e^{-\frac{d \sigma p}{k_{\mathrm{B}} T}}$,

where $G_{0, \lambda}$ denotes the electrical conductance in vacuum, $k_{\mathrm{B}}$ is the Boltzmann constant and $T$ is the temperature (Reif, 1976).

\section{Design and realization}

One possibility to handle the difficulty of measuring low currents is to use energy sources like ultraviolet light to increase 


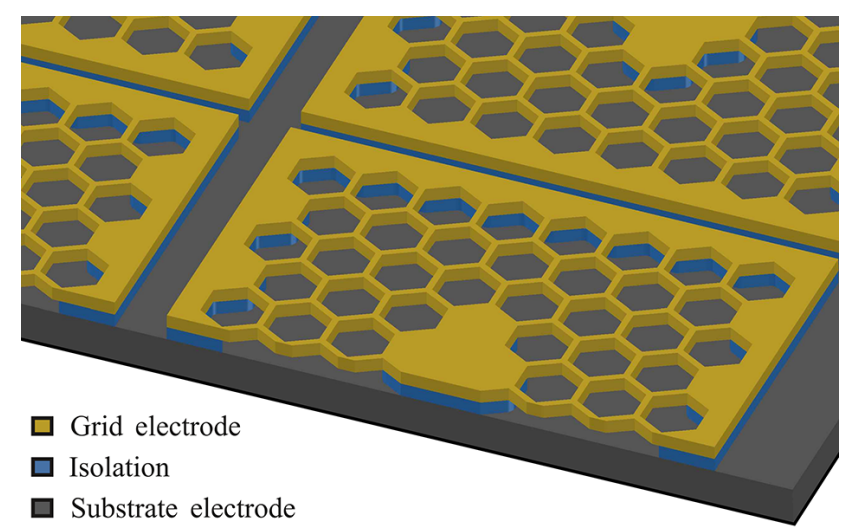

Figure 1. Design study of a chip to measure the conductivity of gaseous substances: levitating honeycombed grid electrode and substrate electrode are isolated by spacers made of $\mathrm{SiO}_{2}$.

the rate of ionized particles within the gas. This is done in conventional gas sensors like photo ionization detectors for example. In contrast, the idea discussed in this article is about optimizing the dimensions of the gas volume to increase the conductance. In the following, the design and realization of the sensor chip will be presented and the experimental setup will be described.

\subsection{Design and principle of operation}

An increased conductance can be obtained by using a gas volume with a large area $A$ perpendicular to the electric field and a short distance $d$ between the electrodes. A design study for a chip that complies with these requirements is shown in Fig. 1. The sensor chip consists of two electrodes, namely the silicon substrate and a levitating grid. The hexagonal grid made of titanium nitride (TiN) is supported by abutments made of silicon dioxide $\left(\mathrm{SiO}_{2}\right)$. The honeycombed structure of the grid originates from an optimization of the mechanical strength of freely suspended grids (Kallis et al., 2014). The wide, honeycombed vent holes of the grid provide fast gas exchange and therefore a fast response time. The final layout of the chip results in a grid of $5 \mathrm{~mm} \times 5 \mathrm{~mm}$ and a length of the gas volume (distance between grid and substrate) of $300 \mathrm{~nm}$.

\subsection{Fabrication of the sensor chip}

The sensor chip was manufactured in planar technology on a p-doped silicon substrate. A flow-chart with cross-section illustrations of the fabrication process is displayed in Fig. 2. The first step was the plasma enhanced chemical vapor deposition (PECVD) of a $300 \mathrm{~nm} \mathrm{SiO} 2$ layer. This layer was coated with $300 \mathrm{~nm}$ TiN, deposited with reactive magnetron sputtering. The honeycombed grid structures were patterned by photo-lithography (with MicroChemicals AZ MIR 701) and structured by reactive ion etching with chlorine gas $\left(\mathrm{Cl}_{2}\right)$, (a)

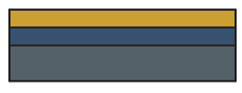

(d)

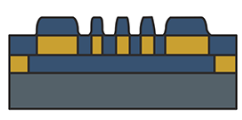

$\mathrm{SiO}_{2}$

Si-substrate

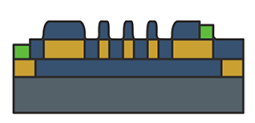

(b)

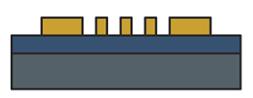

(e)

TiN/Ti/Al (c)

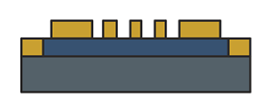

(f)

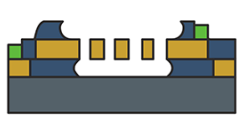

Figure 2. Cross sections of the fabrication using planar technology. (a) Deposition of $\mathrm{SiO}_{2}$ and $\mathrm{TiN}$, (b) structuring with reactive ion etching, (c) contact of the substrate, (d) isolation with plasma enhanced chemical vapor deposition (PECVD) silicon dioxide, (e) bond pads made of TiN/Ti/Al and (f) final suspension of the levitating grid.

methane $\left(\mathrm{CH}_{4}\right)$ and silicon tetrachloride $\left(\mathrm{SiCl}_{4}\right)$ at $40^{\circ} \mathrm{C}$ (see Fig. 2b).

To provide good electrical contact to the substrate, TiN was deposited on the marginal parts of the chip. Therefore, a photo-lithography with image reversal photoresist (MicroChemicals AZ 5214 E) was done in positive mode. Silicon dioxide was removed using wet etching with buffered oxide etch (BOE) followed by the deposition of TiN with reactive magnetron sputtering. For structuring the TiN layer, a lift-off procedure was performed with acetone at $40^{\circ} \mathrm{C}$ supported by sonication (see Fig. 2c). Afterwards, another $300 \mathrm{~nm}$ thick layer of silicon dioxide was deposited to avoid short circuits between substrate- and grid-electrode (Fig. 2d).

The next process steps are needed to build contact pads on the grid and on the titanium nitride margin. This is done because electrical contact to the chip will be provided by ultrasonic bonding later on, when the chip is mounted on a circuit board. Figure 2e shows the contact pads on the grid and the TiN margin. Photo-lithography with image reversal photoresist (MicroChemicals AZ $5214 \mathrm{E}$ ) was done in positive mode, before the silicon dioxide layer was removed with BOE. The contact pads are made of titanium nitride, titanium (Ti) and aluminum $(\mathrm{Al})$, deposited with reactive magnetron sputtering and structured by lift-off.

The final step to obtain the levitation of the grid (Fig. 2f) is done by wet etching with $\mathrm{BOE}$ after another photolithography with MicroChemicals AZ 5214 E in positive mode. Figure 3 shows the scanning electron micrograph of a levitating grid. In the cross-sectional view it is possible to evaluate the removal of the silicon dioxide sacrificial layer under the grid. The bars have a width of approximately $800 \mathrm{~nm}$ and the diameter of the holes is about $6 \mu \mathrm{m}$. On the brink of the grid the abutments of $\mathrm{SiO}_{2}$ are visible underneath. 


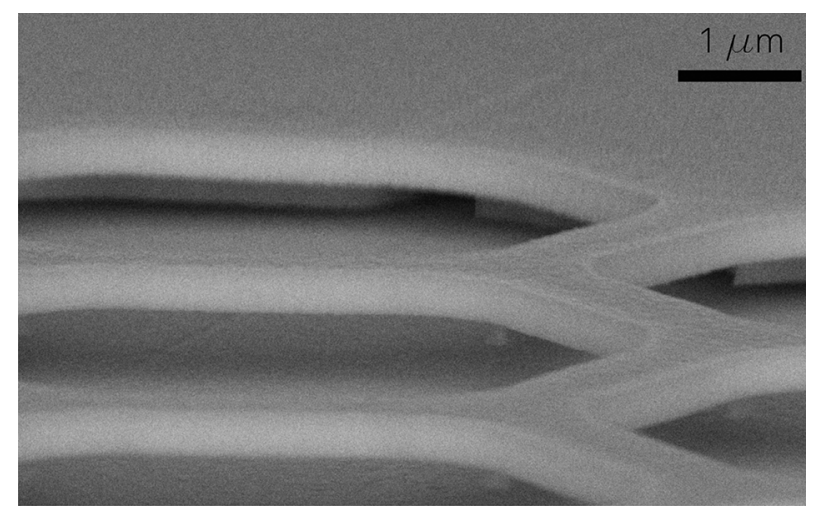

Figure 3. Cross-sectional scanning electron micrograph of a microstructured, levitating grid made of titanium nitride based on silicon dioxide abutments.

\subsection{Experimental setup}

For the electrical characterization, the chip with a size of $8 \mathrm{~mm} \times 8 \mathrm{~mm}$ was attached on a circuit board. Electrical contact was provided by ultrasonic wire bonding. Inside the vacuum chamber the circuit board was soldered to the measuring lines. The vacuum chamber was specially designed to offer the possibility of measuring low currents at different vacuum pressures. It is placed inside a measurement cabinet to avoid vibration and disturbing noise. The pump is a Pfeiffer Vacuum TMU 065 turbo-molecular pump, the pressure was measured with a Pfeiffer Vacuum MPT 100 gauge. Additionally there is a throttle valve to control the flow of gaseous substances into the chamber. To run the electrical measurements an Agilent 4156C precision semiconductor parameter analyzer was used. This setup provides the measurement of currents between femtoamperes and milliamperes at pressures between $1 \times 10^{-6}$ and 1000 mbar. In sweep measurements at different pressures, the current $I$ is monitored while the voltage $U_{\mathrm{G}}$ is swept. These current-voltage characteristics supply the information that is needed to determine the electrical conductance $G$ as a function of pressure.

To evaluate the advantage of the sensor chip over a macroscopic setup concerning the measurement of conductivity due to optimized dimensions, a reference experiment with grids on circuit boards at a distance of approximately $1 \mathrm{~mm}$ was passed. The area of these grids perpendicular to the electric field is approximately the same as the area of the grid on the sensor chip. Subsequently, similar measurements were carried out with the sensor chip, where the gap between the electrodes is reduced by a factor of $3 \times 10^{-4}$ compared to the dimensions of the reference setup.

\section{Results}

In the following, the measurements with both experimental setups will be presented and analyzed. Determination and

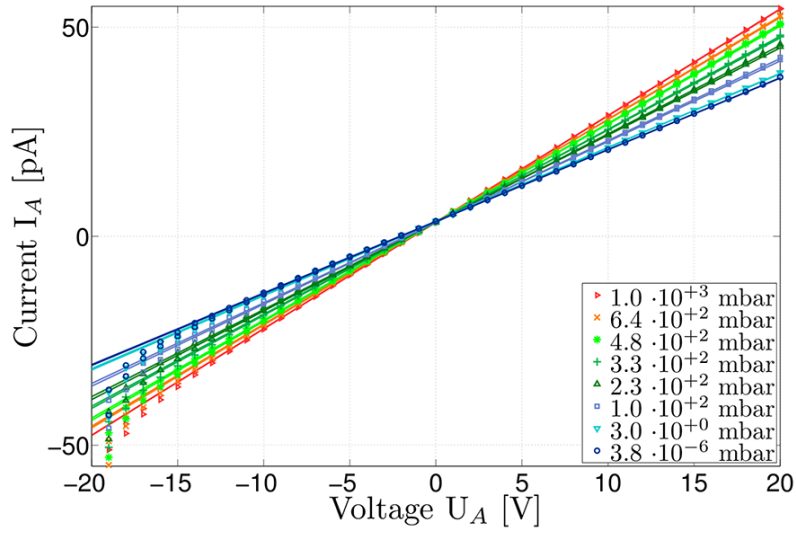

Figure 4. Current-voltage characteristics with electrodes at a distance of $1 \mathrm{~mm}$ at different pressures. The solid lines represent simple linear regressions.

quantitative analysis of the conductance-pressure characteristic of the reference setup with a gap of $1 \mathrm{~mm}$ between cathode and anode will be described. Subsequently, analogues measurements with the sensor chip with a gap of $300 \mathrm{~nm}$ are presented and compared with the previous results.

\subsection{Current-voltage characteristics with $1 \mathrm{~mm}$ gap}

Eight out of 13 sweep measurements with electrodes $1 \mathrm{~mm}$ apart at different pressures are shown in Fig. 4. Measurements were executed at pressures between $3.8 \times 10^{-6}$ and 1000 mbar. Grid voltage was swept from -20 to $20 \mathrm{~V}$ twice at each pressure to verify reproducibility. The current is a linear function depending of the applied voltage (the electric field) and depends on the pressure. Current-voltage characteristics measured at the same pressure hardly differ from each other. Referring to Eq. (12), an electric field of about $20 \mathrm{~V} \mathrm{~mm}^{-1}$ is estimated for an applied voltage of $20 \mathrm{~V}$. The linearity of the characteristic curves is consistent with the theoretic background presented in Sect. 2 as the experimental setup complies with the requirements for the linear approximations in Eqs. (6) and (7).

For further evaluation, simple linear regression was used. The slope of the fitted lines is equal to the electrical conductance $G$ according to Eq. (1). The mean value of the slope with error bars calculated from the two measurements at each pressure is displayed in Fig. 5 as a function of pressure. The error bars are nearly invisible as the standard deviation is very small. The solid line in Fig. 5 results from non-linear curve fitting with the "trust region reflective" algorithm to the function

$G_{1 \mathrm{~mm}}(p)=m \sqrt{p / p_{0}}+b$,

with $p_{0}=1 \mathrm{mbar}$ and the fitting parameters $m=$ $(27.6 \pm 0.8) \mathrm{fS}$ (femto siemens) and $b=(1.71 \pm 0.01) \mathrm{pS}$ referring to Eq. (11) for ions ionized in the gas volume at lower pressures. 


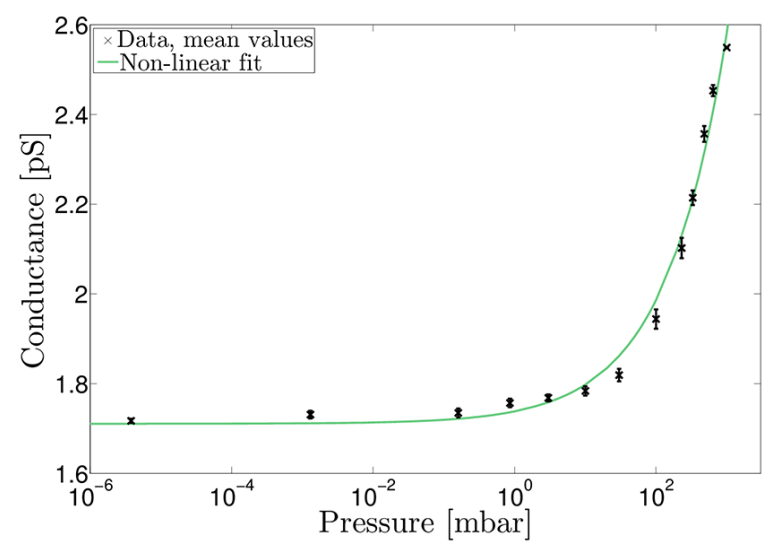

Figure 5. The electrical conductance $G$ as a function of pressure with electrodes at a distance of $1 \mathrm{~mm}$. The values for the electrical conductance result from linear regression of the sweep measurements. The solid line is a non-linear fit to the data according to Eq. (15).

The results of data analysis correspond to the expectation that the electrical conductance is a function of the charge carrier density and therefore will be a function of the pressure. The electrical conductance $G$ is increasing with rising pressure in particular in the range above 1 mbar. This might be due to the fact that the electron's mean free path becomes smaller than the distance between the electrodes of $1 \mathrm{~mm}$ for pressures higher than 0.4 mbar. This could result in a larger number of collisions and therefore more charge carriers in the volume due to ionization. Obviously the fitted curve is in quite good agreement with the data. For mercury vapor, Eq. (10) applies up to a pressure of 3 mbar, but it seems that this limit shifts to higher pressures for air. It is likely that the conductance mechanism is due to ionization in the gas volume with a pressure-dependent charge carrier mobility but a final conclusion of this question is not possible at the moment as the mobility of ions in strong electric fields has not been entirely understood and is still of interest in current research (Liu et al., 2015).

\subsection{Current-voltage characteristics with $300 \mathrm{~nm}$ gap}

Seven examples for sweep measurements at different pressures with the sensor chip are displayed in Fig. 6. The gap between the electrodes is $300 \mathrm{~nm}$. The grid voltage was swept from -1 to $1 \mathrm{~V}$. It is worth mentioning that the electric field on the chip is more than 2 orders of magnitude higher than the electric field applied during the reference measurements due to the small distance between substrate and grid. According to Eq. (12), an electric field of about $3 \mathrm{kV} \mathrm{mm}^{-1}$ can be estimated when a voltage of $1 \mathrm{~V}$ is applied to the grid. Measurements were carried out at pressures between $5.0 \times 10^{-6}$ and 1000 mbar. The measurements were repeated once or twice at each pressure to ensure reproducibility of the results. The characteristics differ clearly from the ohmic behavior

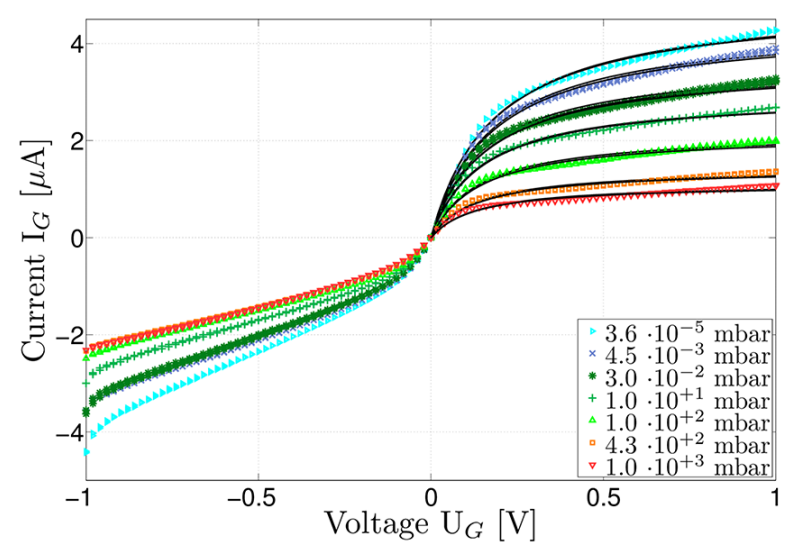

Figure 6. Current-voltage characteristics with the sensor chip (electrodes at a distance of $300 \mathrm{~nm}$ ) at different pressures. The solid black lines are fitted curves.

observed in the reference measurements (see Fig. 4), which is reasonable because the prerequisites for a linear approach do not apply due to the large electric fields. For small electric fields, there is a residual current region where an ohmic behavior may be estimated. For higher values of the electric field, the current approaches a saturation current.

For further evaluation, a curve of the form

$I=\frac{c_{1} U}{c_{2} U+c_{3}}$

was fitted to the current-voltage characteristics in the positive range of voltage (solid black lines in Fig. 6) with the fitting parameters $c_{1}, c_{2}$ and $c_{3}$. This curve describes the current-voltage characteristics for both special cases discussed in Sect. 2, Eqs. (3) and (5). For curve fitting, the "trust region reflective" algorithm was used. The values for the slope in the ohmic region with small electric fields,

$G=\frac{c_{1}}{c_{3}}$,

correspond to the conductance $G$ in Eq. (1) and should show a dependence on pressure that is similar to the linear approximations in Eqs. (6) and (7) if one of the two special cases applies. The mean values of the results of the curve fitting for measurements at different pressures are displayed in Fig. 7. The conductance drops from more than $25 \mu \mathrm{S}$ at low pressures to less than $10 \mu \mathrm{S}$ at atmospheric pressure.

The decrement of conduction with rising pressure points to the fact that the current between substrate and grid does not result from ionization in the gas in contrast to the results deduced in Sect. 4.1. The emission of electrons from the substrate seems to be more likely instead. However, the dependence of the conductance on the pressure cannot be explained by Eq. (6). This is reasonable as the electron's mean free path is larger than $300 \mathrm{~nm}$ for pressures below 1400 mbar and Eqs. (8) to (10) do not apply. The solid line 


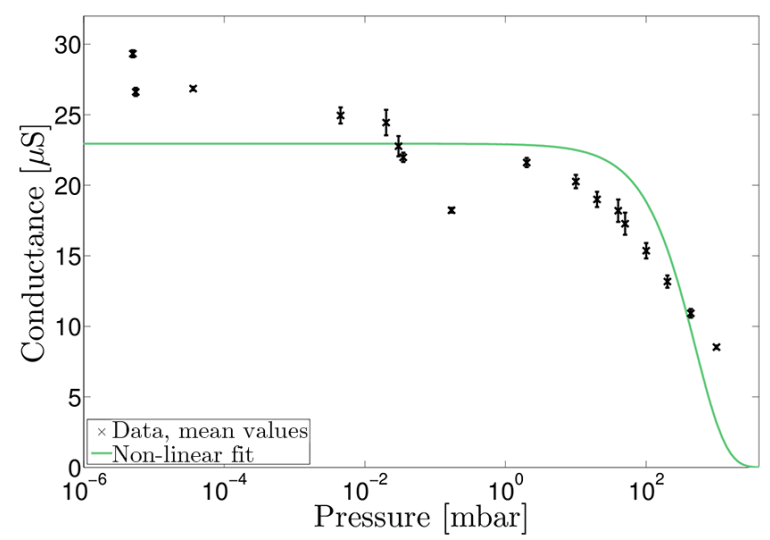

Figure 7. The electrical conductance $G$ as a function of pressure with electrodes at a distance of $300 \mathrm{~nm}$. The values for the electrical conductance result from the fitted curves of the sweep measurements (cf. Eqs. 16 and 17). The solid line is a non-linear fit to the data according to Eq. (14).

in Fig. 7 is the outcome of fitting Eq. (14) to the mean values of the conductance. As a result, the scattering cross section $\sigma=(2.7 \pm 0.8) \times 10^{-19} \mathrm{~m}^{2}$ can be calculated from the parameters of the fitted curve, given by

$G_{300 \mathrm{~nm}}=G_{0} e^{-p / p_{0}}$,

referring to Eq. (14) with the fitting parameters $G_{0}=$ $(23 \pm 1) \mu \mathrm{S}, p_{0}=(510 \pm 150) \mathrm{mbar}$ and a distance between grid and substrate of $d=300 \mathrm{~nm}$. The value for the cross section is in the range of the values of electrons with a kinetic energy of $1 \mathrm{eV}$ colliding with oxygen and nitrogen $\left(\sigma_{\mathrm{O}_{2}}=5.97 \times 10^{-20} \mathrm{~m}^{2}, \sigma_{\mathrm{N}_{2}}=1 \times 10^{-19} \mathrm{~m}^{2}\right)$, reported in literature (Itikawa et al., 1986; Itikawa, 2009). Even though the shape of the curve does not fit the data very well, and the standard deviation of the fitting parameter $p_{0}$ is rather high, the deceleration of electrons due to collisions with gas molecules is likely to be the reason for decreasing conductances with rising pressures. However, there have to be further mechanisms of conductivity not considered yet.

Contrary to the measurements with a gap of $1 \mathrm{~mm}$, the current increases on the sensor chip when the pressure drops and the currents are thereabout 5 orders of magnitude larger than in the reference measurements. This cannot simply be explained by a reduced distance between the electrodes. Both Eqs. (6) and (7) indicate that the current is proportional to $1 / d$ and a rise of 3 orders of magnitude in the current would be sensible. Thus, a supplemental effect that causes higher currents has to be taken into consideration.

\section{Discussion}

This work is the first research testing conductance measurements as a measurement principle for MEMS-type vacuum sensors. With regard to the question of whether this con- cept is promising for the development of vacuum sensors, the findings presented above have revealed that the sensitivity, defined as $\mathrm{d} G / \mathrm{d} l g(p)$ (Sun et al., 2013), is approximately $1 \mu \mathrm{S}$ per decade for pressures smaller than 1 mbar. For this pressure range, the sensitivity of the sensor chip is low but it is not zero as predicted in Eq. (14). The proposed model explains one aspect of the results but cannot explain the overall behavior of the sensor chip yet. Further research on the causes of the decreasing conductance in this pressure range could also reveal possibilities to improve the sensitivity. The fabricated sensor chip shows a good sensitivity of approximately $12 \mu \mathrm{S}$ per decade from 1 to $1000 \mathrm{mbar}$. Compared to other sensor principles, this is a narrow range of pressure as there are already many suggestions for MEMS-type vacuum sensors with a pressure range over 8 orders of magnitude. However, most of the sensors presented in literature work on sensor principles that have been improved over years (Randjelović et al., 2014). The analysis of the measurements indicates that the sensitive range of the sensor could be adapted by varying the distance between substrate and grid. Previous studies have shown that the sensitivity of ion gauges, both conventional and field-emission-based vacuum gauges, is determined by the shape, dimensions and the material of the electrodes (Wilfert and Edelmann, 2012). In the future, further research should be carried out to examine if the variation of design and materials of the sensor chip first presented in this article will allow the extension of the sensitive range towards lower pressures.

Knowing that low current measurements are a challenge for this type of sensor, this study aimed to reach higher currents by miniaturization of the sensor. The results show clearly that the increase in current exceeds the expectations and that this effect cannot be explained by a reduced distance between the electrodes. One possible explanation is that field emission due to inhomogeneities of the electric field is the reason for this discrepancy. In previous studies, field emission structures have been used as electron sources. However, these studies are predominantly focused on tip arrays and did not examine the emission from wider surfaces (Wen et al., 2007). Finite element analysis of the electric fields and the investigation of electron emission from the substrate could lead to a better understanding of the physical mechanisms on the sensor chip. Overall, it could be shown that the development of the sensor chip led to an amplification of the pressure-dependent measuring signals, which exceeded expectations. It is very likely that simple multimeters or integrated readout circuits are sufficient to measure the signals. This would mean a significant reduction of the measuring effort.

\section{Conclusions}

In this research, a new approach for measuring low pressures with a MEMS sensor chip has been demonstrated. The the- 
oretical background of electrical conduction mechanisms in gases has been summarized. Design, manufacturing and electrical characterization of the sensor chip have been presented, analyzed and discussed. The electrical characterization of the sensor chip showed a pressure-dependence of the conductivity from $5 \times 10^{-6}$ to 1000 mbar. Currents in the range of microamperes offer a reduction in the amount of measurement instrumentation. In summary, the MEMS sensor discussed here is regarded to be a promising concept for simple and competitive microscopic devices for low pressure measurements.

Data availability. The data presented in this article are stored in an internal system according to the guidelines of the DFG.

Competing interests. The authors declare that they have no conflict of interest.

Special issue statement. This article is part of the special issue "Sensors and Measurement Systems 2016". It is a result of the 18. GMA/ITG Fachtagung Sensoren und Messsysteme 2016, Nuremberg, Germany, 10-11 May 2016.

Acknowledgements. The authors would like to thank the Ministry of Education and Research for financial support and Karola Kolander, Marko Kremer, Ulrike Lippe and Achim Wiggershaus for their technical and administrative support.

Edited by: Andreas Nocke

Reviewed by: two anonymous referees

\section{References}

Dankovic, T., Punchihewa, K. A. G., Zaker, E., Farid, S., Habibimehr, P., Feinerman, A., and Bustaa, H.: Extension of operating range towards lower pressures of MEMS-based thermal vacuum gauges by laser-induced heating, Procedia Engineer., 47, 1243-1246, https://doi.org/10.1016/j.proeng.2012.09.378, 2012.

Górecka-Drzazga, A.: Miniature and MEMS-type vacuum sensors and pumps, Vacuum, 83, 1419-1426, https://doi.org/10.1016/j.vacuum.2009.05.003, 2009.

Itikawa, Y.: Cross Sections for Electron Collisions with Oxygen Molecules, J. Phys. Chem. Ref. Data, 38, 1-20, https://doi.org/10.1063/1.3025886, 2009.

Itikawa, Y., Hayashi, M., Ichimura, A., Onda, K., Sakimoto, K., Takayanagi, K., Nakamura, M., Nishimura, H., and Takayanagi, T.: Cross Sections for Collisions of Electrons and Photons with Nitrogen Molecules, J. Phys. Chem. Ref. Data, 15, 985-1010, 1986.

Kallis, K., Dietz, D., Subasi, E., Müller, M., Kontis, C., and Zimmer, C.: Design, simulation, fabrication and characterization of nano-scaled acceleration grids, Microelectron. Eng., 121, 118121, https://doi.org/10.1016/j.mee.2014.04.036, 2014.
Lee, H.-C. and Huang, R.-J.: A novel field emission array pressure sensor, in: Solid-State Sensors and Actuators, 1991, Digest of Technical Papers, 241-244, https://doi.org/10.1109/SENSOR.1991.148847, 1991.

Liu, Y., Huang, S., and Zhu, L.: Influence of humidity and air pressure on the ion mobility based on drift tube method, CSEE J. Power Energy Syst., 1, 37-41, https://doi.org/10.17775/CSEEJPES.2015.00033, 2015.

Randjelović, D. V., Frantlović, M. P., Miljković, B. L., Popović, B. M., and Jakšić, Z. S.: Intelligent thermal vacuum sensors based on multipurpose thermopile MEMS chips, Vacuum, 101, 118-124, https://doi.org/10.1016/j.vacuum.2013.07.044, 2014.

Reif, F.: Grundlagen der Physikalischen Statistik und der Physik der Wärme, Walter de Gruyter, Berlin, New York, 541-554, 1976.

Rutherford, P. H. and Goldston, R. J.: Introduction to plasma physics, Inst. of Physics Publ., Bristol, 3-7, 1995.

Sun, X., Xu, D., Xiong, B., Wu, G., and Wang, Y.: A wide measurement pressure range CMOS-MEMS based integrated thermopile vacuum gauge with an XeF2 dryetching process, Sensor. Actuat. A-Phys., 201, 428-433, https://doi.org/10.1016/j.sna.2013.07.020, 2013.

TEKTRONIX Inc.: Low Current Measurements, in: Low Level Measurements Handbook, 7th Edn., 2.15-2.35, 2013.

Tenholte, D., Kurth, S., Geßner, T., and Dötzel, W.: A MEMS friction vacuum gauge suitable for high temperature environment, Sensor. Actuat. A-Phys., 142, 166-172, https://doi.org/10.1016/j.sna.2007.05.031, 2008.

Völklein, F. and Meier, A.: Microstructured vacuum gauges and their future perspectives, Vacuum, 82, 420-430, https://doi.org/10.1016/j.vacuum.2007.08.001, 2007.

Wang, X., Liu, C., Zhang, Z., Liu, S., and Luo, X.: A micromachined Pirani gauge for vacuum measurement of ultra-small sized vacuum packaging, Sensor. Actuat. A-Phys., 161, 108-113, https://doi.org/10.1016/j.sna.2010.04.034, 2010.

Wasserrab, T.: Gaselektronik I, chapter 4.2.2 "Die Ladungsträgerbeweglichkeit im elektrischen Feld",Bibliographisches Institut Zürich, Zürich, Switzerland, 158-165, 1971.

Wen, W., Wang, L., Gao, J., and Sun, D.: Studies on MEMS Vacuum Sensor Based on Field Emission of Silicon Tips Array, in: 2007 2nd IEEE International Conference on Nano/Micro Engineered and Molecular Systems, 199-202, https://doi.org/10.1109/NEMS.2007.352261, 2007.

Wiesemann, K.: Einführung in die Gaselektronik, chapter 2.4 "Deutung der Charakteristik einer unselbständigen Entladung”, B. G. Teubner Stuttgard, 20-25, 1976.

Wilfert, S. and Edelmann, C.: Field emitterbased vacuum sensors, Vacuum, 86, 556-571, https://doi.org/10.1016/j.vacuum.2011.08.008, 2012.

Zhang, F., Tang, Z., Yu, J., and Jin, R.: A micro-Pirani vacuum gauge based on micro-hotplate technology, Sensor. Actuat. APhys., 126, 300-305, https://doi.org/10.1016/j.sna.2005.10.016, 2006.

Zhou, C.-Y., Su, H., Mao, R.-S., Dong, C.-F., Qian, Y., and Kong, J.: An accurate low current measurement circuit for heavy iron beam current monitor, Nucl. Instrum. Meth. B, 280, 84-87, https://doi.org/10.1016/j.nimb.2012.01.033, 2012. 